\title{
ZENTRALE UND DEZENTRALE GESTALTUNG DES LEITUNSSYSTEMS IN BETRIEBEN
}

\author{
Doç. Dr. Tamer MÜFTÜOǴLU
}

\section{A) Zentralisation und Dezentralisation}

\section{Die Begriffe der Zentralisation und Dezentralisation}

Zentralisation und Dezentralisation stellen die Grundprinzipien der Aufgabensynthese dar. In der Literatur aber besteht keine einheitliche Begriffsbestimmung über diese Prinzipien. Man kann diese Auffassungen in zwei Gruppen unterscheiden: einmal behandelt man Zentralisation und Dezentralisation als spezielles Problem der Zuordnung der Entscheidungsund Leitungsaufgaben und zweitens als allgemeines Problem der Zuordnung von Aufgaben. ${ }^{1}$

Fayol definiert die Zentralisation als die Konzentration der Entscheidungen von wichtigen Fragen in gewissen Zentren, um damit der Bedeutung dieser Entscheidungen Rechnung getragen wird, sowie die Dezentralisation als die Verminderung der Bedeutung dieser Zentren zugunsten der Untergebenen. ${ }^{2}$

Bei Davis ist die Dezentralisation eine Art von Verantwortungsübertragung, die sich aus dem Delegationsproze $\beta$ ergibt. ${ }^{2}$

Simon unterscheidet zuerst zwei Arten von Zèntralisation. "Man kann die Entscheidungsgewalten zentralisieren, in dem man allgemeine Vorschriften benutzt, die das Ermessen der Untergebanen eingrenzen. Man kann Entscheidungsgewalten aber auch zentralisieren, in dem man dem. Untergebenen die tatsächliche Entscheidungsgewalt aus der Hand nimmt."3 Da diese beiden Arten den Ermessensspielraum der Untergebenen eingrenzen, gelten sie als Zentralisation.

\footnotetext{
1 Bleicher: Zur Zentralisation und Dezentralisation des Entscheidungsprozesses in der Unternehmungsorganisation. Festschrift für E. Kosiol s. 110.

2. Stroudakis, P.: Organisation der Unternehmungsführung, s. 109, 110.

3 Simon: Verwaltungshandeln, s. 150.
} 
Stroudakis will besonders die Unterscheidung von Dezentralisation und Delegation hervorheben. "Das unterscheidende Merkmal der Dezentralisation von der Delegation besteht im wesentlichen in der fehlenden Verpflichtung des untergeordneten Funktionssubjektes zur Rechenschaft." Der Delegationsproze $\beta$ erfolgt auch bei der Zentralisation, aber hier wird die Autorität nicht unbedingt und Verantwortung auf keinen Fall delegiert.

Kosiol behandelt Zentralisation und Dezentralisation als ein allgemeines Problem der Zuordnung von Aufgaben. "Zentralisation und Dezentralisation sind zwei Prinzipien, die sich gesenseitig bedingen. Sie geben die Richtungen an, in der synthetisch die Bildung einer Aufgabe erfolgt. Zentralisation bedeutet die Zusammenfassung und Dezentralisation die Trennung von Teilaufgaben, die hinsichtlich eines Merkmals gleichartig sind."s Auf dem Gedanken Kosiols aufbauend, definiert Bleicher die Zentralisation als ein Streben zu einem Zentrum hin und die Dezentralisation als ein Streben von einem Zentrum fort. Sie beziehen sich auf Handlungen, die in verschiedenen Richtungen vorgenommen werden können und geben die Richtung dieser Handlungen an.

\section{Das Wesen der Zentralisation und Dezentralisation}

Nach Kosiol sind die Objekte der Zentralisation und Dezentralisation die Teilaufgaben, die zentralisiert bzw. dezentralisiert werden, Ziele sind Stellen und Abteilungen, in denen Zentralisation bzw. Dezentralisation erfolgt. Bei einer zentralen Gestaltung der Lagerung von Werkstoffen werden Teilaufgaben, die mit dieser Lagerung verbunden sind, in einer Abteilung zusammengefaßt. Bei einer dezentralen Gestaltung dagegen werden diese Teilaufgaben denjenigen Stellen oder Abteilungen, denen bestimmtue Lageraufgaben zugewiesen werden, zugeteilt. Von den aufgabenanalytischen Merkmalen und den übrigen Bestimmungselementen einer Aufgabe ergeben sich folgende idealtypischen Gruppen von Zentralisations- bzw. Dezentralisationsformen: persönliche Zentraisation, sachliche Zentralisation, formale Zentralisation, Mittelzentralisation, Raum- und Zeitzentralisation.

Wie auch vom obigen Lagerbeispiel zu verstehen ist, hat die Zentralisation und Dezentralisation elinen graduellen Charakter. Acker versucht diesen Grad sogar zahlenmäßig zu ermitteln. ${ }^{7}$ Er geht davon aus, daß jede

4 Stroudakis, P.: Organisation der Unternehmungsführung, s. 110.

s Kosiol, E.: Organisation der Unternehmung, s. 81.

- Kosiol, E.: a.a.O., s. 81, 82.

7 Acker, H.: Die organisatorische Stellengliederung im Betrieb, s. 122. 
Tätigkeit folgende Merkmale hat: Objekt, Verrichtung, Werkzeug, Subjekt, Raum, Zeit und Ziel. Diese Merkmale könnèn in verschiedenen Genauigkeitsgraden bestimmt werden. Wenn man die Merkmale und ihre Genauigkeitsgrade einer leitenden Tätigkeit multipliziert, so erhält man eine Zahl, die angibt, wieviele Bestimmungen überhaupt nötig sind, um diese Tätigkeit zu bestimmen. Die Anzahl dieser Tätigkeiten kann auf verschiedene Stufen des organisatorischen Aufbaus einer Unternehmung verteilt werden. Im Extremfall können einmal alle Bestimmungen von oberster Stufe und zum anderen alle Bestimmungen von unterster Stufe vor:genommen werden. Theoretisch ist das erste Extrem nur in einer zweistufigen Organisation, das zweite überhaupt nicht möglich. Zwischen diesen Extremen liegen aber die möglichen Lösungen. Je größer der Anteil der obersten Instanzen ist, desto größer ist auch der Zetralisationsgrad und umgekehrt.

Auch Simon's Versuch, die Abhängigkeit der Zentralisation und Dezentralisation von der Prüfungsart, die im Betrieb àngewendet wird, nachzuweisen, wird hier dargelegt. "Wenn das Prüfungsverfahren zur Korrektur individueller Entscheidungen benutzt wird, führt es zur Zentralisierung und $\mathrm{zu}$ einer tatsächlichen Übertragung von Entscheidungen. Zweitens, wenn es benutzt wird, um aufzudecken, wo der Untergebene einer zusätzlichen Leitung bedarf, führt es wiederum zur Zentralisierung, weil immer mehr umfassande Vorschriften und Anweisungen ergehen müssen, die den Ermessensspielraum des Untergebenen einschränken. Wenn es drittens benutzt wird, um aufzudecken, wo die eigenen Mittel des Untergebenen verstärkt werden müessen, führt es zur Dezentralisierung. Alle drei Elemente können in verschiedenen Umfange vorhanden sein und sind gewöhnlich auch gleichzeitig vorhanden."

Wenn wir vom soziologischen Aspekt der Zentralisation und der Dezentralisation ausgehen, so können wir sagen, da $\beta$ das Eigentum der Individien eine ökonomische Dezentralisation des Gesamteigentums darstellt. "So wird in der Gesellschaft zugunsten der Individien dezentralisiert, die selbst zentralisieren." Die Menschen haben also eine Neigung zur Zentralisation, und wegen dieser Neigung der Individien erfolgt in einer freien Gesellschaft der Dezentralisationsprozeß. "Wenn tatsächlich der Mensch eine solche Neigung zur Zentralisation nicht hätte (von Eigentum, Macht, Autorität), so würde der Staat das Eigentum, die Macht, die Autorität bei sich zentralisieren, die Individien könnten keine Initiative mehr entfalten, ihr Leben müßte verkümmern."’.

8 Simon: a.a.O., s. 150.

9 Stratoudakis: a.a.O., s. 112. 
B) Die Zentralisation und Dezentralisation von Entscheidungen

\section{Die Trennung von Entscheidung und Ausführung}

$\mathrm{Ab}$ einer bestimmten Größe kann die Unternehmung ihre Aufgaben ohne Anwendung des Prinzips der interpersonalen Arbeitsteilung nicht mehr bewältigen. So wird es notwendig, den ausführenden Aufgabenträgern einige Entscheidungen abzunehmen und diese in bestimmten Stellen zu zentralisieren. Besonders "Es sind die Entscheidungen, die von keinem anderen ausführenden Aufgabenträger mehr gelöst werden können. Die Aufgabe der Zielsetzung für den Arbeitsproze $\beta$, der interpersonalen Arbeitsverteilung und der Sicherstellung des Arbeitsergebnisses durch Abstimmungsmaßnahmen."

Auf Grund dieser Trennung von Entscheidung und Ausführung werden diese zentralisierten Stellen Fremdentscheidungen treffen. Sie sind also im Sinne Kosiols übergeordnete Stellen bzw. Leitungsstellen. Durch die Gleichordnung der Entscheidungsaufgaben mit den Ausführungsaufgaben zu sogenannten Selbstentscheideungen, werden die ausführenden Aufgabenträger einen Ermessensspielraum besitzen.

\section{Die Verbindunsprobléme von Entscheidung und Ausführung}

\section{Die natürliche Enheit von Entscheidung und Ausführung}

Wenn die Entscheidungs- und Ausführungsaufgaben auf denselben Aufgabenträger fallen, dann existiert eine natürliche Einheit von Zielsetzung und Zielerreiehung. Das ist, wie oben dargelegt wurde, in der Gleichordnung von Entscheidungs- und Ausführungsaufgaben der'Fall. Hier, "zwischen Entscheidung und Ausführung ergeben sich keine Einigungsprobleme. Hinzu kommt der motivierende Umstand, da $\beta$ Aufgabenträger, die selbst eine Entscheidung geplant haben, sich ihrer Verwirklichung stärker verbunden fühlen als dies im allgemeinen bei der Ausführung fremder Entscheidungen der Fall ist."11

\section{Die künstliche Einheit von Entscheidung und Ausführung}

Durch die persönliche Trennung von Entscheidung und Ausführung entsteht ein Einungsproblem dieser beiden Aufgabenarten .Hier geht es darum, die natürliche Einheit von Entscheidung und Ausführung durch eine künstliche Einheit zu substituieren. Diese Substitution ist durch die

10 Bleicher: Kunt, a.a.O., s. 112.

11 Bleicher: a.a.O., s. 113. 
Beziehungen zwischen der Leitung und Ausführung zu erreichen. Diese Beziehungen sind Verantwortungs- und Arbeitsbeziehungen.

a) Verantwortungsbeziehung

Ich zitiere Hasenack: "Wenn Heidegger Verantwortung zu erklären hätte, würde er im Denken seiner semantischen Methode etwa wie folgt vorgehen: In Verantwortung steckt Antwort; Antwort setzt Frage voraus. Die entsprechenden Fragen müssen heißen: Hast du deinen Auftrag erfüllt? Warum hast du dies oder jenes getan, oder unterlassen? Antworte, verantworte dich!"'12

"Werantwortlich sein" auf die Frage "wofür" ist also immer etwas Bestimmtes, möglichst Ümrissenes. Diese Frage "wofür" ist nach dem Gegenstand der Verantwortung ausgerichtet. Die Frage "wovor" oder "vor wem" Verantwortung getragen wird, bildet die Richtung der Verantwortung.

Durch die personale Trennung von Entscheidung und Ausführung wird die Verantwortungsbeziehung zweiseitig. Sie liegt einmal in der Bedeutung des Antwortenmüssens (Verantwortungspflicht) auf die Frage nach der Zielerreichung des Tätigseins und zum anderen im Recht zum Fragen nach der Zielerreichung der Aufgabenerfüllung anderer, weisungsgebundener Personen (Verantwortungsrecht). Verantwortungsrecht ergibt sich aus leitender Tätigkeit. Verantwortungspflicht und Verantwortungsrecht bilden die Verantwortungsbeziehungen im Leitungsverhältnis. ${ }^{13}$

\section{b) Die Arbeitsbeziehung}

Die Verantwortungsbeziehungen bilden die Grundlagen der Arbeitsbeziehung zwischen den Leitungsstellen und Ausführungsstellen. Diese Beziehungen können als Informationsbeziehungen dargelegt werden und sind zweiseitig:

b 1) Der leitende Aufgabenträger braucht Informationen von ausführenden Aufgabenträgern, um seine Entscheidungen richtig treffen zu können. Diese Beziehung erfolgt auf Informationswegen von unten nach oben.

b 2) Der leitende Aufgabenträger gibt seine Entscheidungen als Anordnungen den ausführenden Aufgabenträgern kund. Diese Beziehung erfolgt auf dem Befehlsweg oder Instanzenweg von oben nach unten. ${ }^{\text {14 }}$

12 Hasenack: Die Gestaltung der Eigenverantwortlichkeit in der Untrenéhmung. ZfhF. N. F. 1957, s. 282.

13 Kosiol, E.: a.a.O., s. 105, 106.

14 Bleicher: a.a.O., s. 117. 


\section{Zuordnung von Entscheidungsprozessen}

In der Zuordnung von Entscheidungsprozessen wird versucht, die Objektspanne einer Gleiderungseinheit mit dem Objekt des Entscheidungsprozesses zur Deckung zu bringen.

\section{1. nach dem Umfang der Entscheidungen}

Hier unterscheiden wir mit Kosiol zwischen umgreifenden und übergreifenden Entscheidungen. "Umgreifende Entscheidungen" sind solche, die von vornherein das ganze oder mehrere Bereiche der Unternehmung umfassen - "übergreifende Entscheidungen" sind solche, die aus einem Bereich auf andere hinüberwirken." 15 Wir können hier auch zwischen Ganzheits- und Bereichsentscheidungen unterscheiden.

Umgreifende und Ganzheitsentscheidungen sind eine Angelegenheit der obersten Leitung und damit zu zentralisieren. $O b$ die übergreifenden Entscheidungen zentral oder dezentral zu gestalten sind, hängt von der Relevanz dieser Entscheidungen ab. Die Grenzen, Maßstäbe und Bedingungen dieser Relevanz können nur im praktischen Fall festgelegt werden. Die übergreifenden Entscheidungen von geringer Relevanz und reine Bereichsentscheidungen können dezentral gestaltet werden. Die übergreifenden Entscheidungen von großer Relevanz dagegen sind eine Angelegenheit der obersten Instanzen und also zu zentralisieren.

\section{2. nach der Bedeutung der Entscheidungen}

Hier unterscheiden wir zwischen konstitutiven und situativen Entscheidungen. Konstitutive Entschediungen sind für die Unternehmung von größter Bedeutung, sie sind grundlegende Entscheidungen. Z.B. Entscheidungen über Unternehmungsgegenstand, über Standort, Aufgabenumfang, aufzubringende Mittel, Investitionsplanung u.a. ${ }^{16}$ Diese Entscheidungsprozesse sind ihrer Bedeutung nach zentral zu organisieren.

Die situativen Entscheidungen sind situationsbedingte, laufende Entscheidungen. Ob sie zentral oder dezentral zu organisieren sind, kann nur in konkreten Fällen gesagt werden. Sie sind in manchen Betrieben zentral, in anderen dezentral organisiert, obwohl sie demselben Bereich angehören. Sogar im Lebensbild einer Unternehmung sind sie zu verschiedenen Zeiten unterschiedlich organisiert.

15 Kosiol, E.: a.a.O., s. 121.

16 Bleicher, K.: a.a.O., s. 128. 


\section{Vollkommene und unvollkommene Bedingungen}

Die Vollkommenheit der Arbeitsbeziehungen zwischen Leitung und Ausführung ist für die Zentralisation oder Dezentralisation des Entscheidungsprozesses von größter Bedeutung. Im folgenden wird die Vollkommenheit des Informationswesens und die Wiederholbarkeit der Entscheidungsprozesse sowie ihre Bedeutung für die zentrale oder dezentrale Gestaltung der Entscheidungsaufgaben erläutert.

\section{Vollkommene und unvollkommene Informationen}

Hier ist die Vollkommenheit einmal des Informationsgehalts und einmal der Informationstechnik zu berücksichtigen. Die Vollkommenheit des Informationsgehalts kann als quantitativ (wenig, zuviel oder genügender Informationsgehalt) und qualitativ (guter oder schlechter Informationsgehalt) bestimmt werden.

Die Vollkommenheit der Informationstechnik ist von der Ausrüstung des Informationsweges, - von den Mitteln, die man braucht und von der Art der Informationsübertragung abhängig. In die Einzelheiten des Informationswesens wird hier nicht eingegangen.

\section{Wiederholbarkeit der Entscheidungsprozesse}

Hier kann man zwischen wiederkehrenden und einmaligen Entscheidungsprozessen unterscheiden.

\section{2 ) Wiederkehrende Entscheidungsprozesse}

Diese Entscheidungen. sind wegen ihrer häufigen Wiederholbarkeit vorauszusehen und können damit vorher programmiert werden. Sie sind. Routineentscheidungen. Kosiol nennt sie usuale Entscheidungen.

\section{2 b) einmalige Entscheidungsprozesse}

Diese Entscheidungen können wegen ihrer mangelnden Wiederholbarkeit und sogar ihrer Einmaligkeit nicht vorher programmiert werden. Sie sind fallweise Entscheidungen und müssen damit in den Bereich unternehmerischer Dispositionen eingeordnet werden. Sie können einmal solche Entscheidungen sein, die sich in' der schöpferischen Eigeninitiative der Leitung ausdrücken und unter Umständen "bahnbrechend Neuland aufschließen". Sie sind novative Entscheidungen (Kosiol). Obwohl manche Entscheidungen nicht die Merkmale novativer Entschediungen besitzen, sind sie einmalige Entscheidungen, weil ihre Wiederholbarkeit fraglich 
ist. Thre Bedeutung für die Unternehmung ist natürlich geringer als rein novative Entscheidungen.

\section{Zentralisation und Dezentralisation unter vollkommenen und unvollkommenen Bedingungen.}

Die einmaligen Entscheidungen, besonders die novativen Entscheidungen, haben eine natürliche Tendenz zur Zentralisation.

Unter unvollkommenen Bedingungen aber gilt dies nicht ohne weiteres. Wegen des mangelnden Informationswesens ist die zentrale Leitung nicht immer in der Lage, die nötigen Informationen im Hinblick auf ihre Aufgabenerfüllung zu sammeln. Hier ist das Zeitmoment auch zu berücksichtigen; die Leitung kann die Informationen sammeln und auf Grund dieser Informationen eine richtige Entscheidung treffen, aber dies kann zu spät erfolgen. In diesem Fall wird den ausführenden Aufgabenträgern ein weiterer Ermessensspielraum zugestanden und nur die Entscheidungen werden zentralisiert, die aufgrund ihres Umfanges und ihrer Bedeutung an keiner Stelle der Organisation vorgenommen werden können.

Besonders unter den unvollkommenen Bedingungen in einer vielstufigen oder geographisch zu weit verteilten Unternehmung ist diese Gefahr, alle einmaligen Entscheidungen zu zentralisieren, sehr groß. "Trifft dieses trotzdem eine zentrale Entscheidung, so besteht die Gefahr, da $\beta$ diese Entscheidung entweder sachlich unrichtig (wegen unvollkommenen Informationsgehalts) oder zu spät (wegen unvollkommener Informationstechnik, unvollkommener Übermittlungsdauer) getroffen wird: Entscheidungen am grünen Tisch." ${ }^{17}$ Um diese Gefahr auszuschalten, müssen solche Entscheidungen unter unvollkommenen Bedingungen, soweit sie beschränkte Bedeutung für die Existenz der Unternehmung haben, dezentralisiert werden. Bei widerkehrenden Entscheidungen unter vollkommenen Bedingungen besteht eine starke Tendenz zur Dezentralisation. Unter unvollkommenen Bedingungen dagegen besteht in der Praxis eine Tendenz, auch die widerkehrenden Entscheidungen, soweit sie nicht von geringer Relevanz sind, zentral zu organisieren.

\section{Andere Gründe, die die Zentralisation und Dezentralisation von Entscheidungsprozessen bestimmen.}

Simon berücksichtigt auch das Kostendenken. "Es genügt nicht, nur die Genauigkeit der Entscheidung zu bedenken, auch die Kosten müssen erwogen werden. Der Vorgesetzte ist wahrscheinlich höher bezahlt als die

17. Bleicher, K.: a.a.O., s. 110 
Untergebenen. - Es kann sein, da $\beta$ die größere Genauigkeit einer vom Vorgesetzten getroffenen Entscheidung durch einen zu hohen Preis erkauft ist." Er bringt dann gegen die Vorteile der Spezialisierung, deren Folge die Zentralisation ist, außer den Kosten noch folgenden Nachteil vor: "Aber viel inniger und wirksamer sind die Koordinierungsmechanismen des menschlichen Nervensystems. Es ist nicht ganz einfach, einen Faden einzufädeln, wenn eine Person den Faden und eine andere die Nadel hält. Die Aufgabe ist hier, beides an die selbe Stelle zu bringen. Zwischenpersonale Koordinierung erfüllt diese Aufgabe nicht so leicht wie die Koordinierung, die sich aus zweier Händan durch ein und dasselbe menschliche Nervensystem eroibt."18 Die zentrale oder dezentrale Gestaltung hängt auch von der "Freude an Regieren" der Menschen eines Volkes ab. Merkwürdigerweise ist diese "Freude am Regieren" besonders bei Völkern mit knechtischer Veranlagung verbreitet. "Es gibt keine herschfreudigere Regenten, als gewesene Knechte. Es gibt keine befehlsfreudigere Hausfrau als die gewesene Dienstmaged". ${ }^{19}$ Das Bildungs- und Ausbildingsniveau eines Volkes hat auch seinen Einfluß auf Zentralisation und Dezentralisation. In einem Lande, dessen Bevölkerung größtenteils aus Analphabeten besteht, werden die Unternehmungen die Zentralisation vorziehen. Man vertritt auch die Auffassung, da $\beta$ mit dem Einsatz von großen Rechenanlagen eine neue Tendenz zur Zentralisation besteht, weil durch diese Rechenanlagen die Informationen schnell und entscheidungsreif $\mathrm{zu}$ gestalten sind. So werden, behauptet man, die Maßnahmen zur Dezentralisation hinfällig. In den USA vertritt man dagegen die Auffassung, da $\beta$ die Organisationsstruktur von dem Einsatz dieser Rechenanlagen nicht berührt wird. "Diese Rechenanlagen erfüllen lediglich Dienstleistungen, die mit diesen Aufgaben betrauten Abteilungen für die auch weiterhin dezentralisierte Bereiche. Die Rechenanlagen sind vilmehr die gewählte Organisationsstruktur mit dienenden Funktionen einzugliedern.,"20

\section{C) Zentrale oder dezentrale Gestaltung des Leitungssystems}

\section{Schmalenbach's pretiale Betriebslenkung}

"Das Wesen der pretialen Betriebslenkung besteht darin, da $\beta$ die Oberleitung den Nachgeordneten weitgehende Selbständigkeit läßt und nur besonders wesentliche Entscheidungen vorbehält, dafür aber die Leis-

\footnotetext{
18 Simon: a.a.O., s. 152.

13 Schmalenbach, E.: Pretiale Wirtschaftslenkung. Band II. "Pretiale Betriebslenkung" s. 8.

20 Organisation in amerikanischen Unternehmen.- Ergebnisse einer Studienreise in die Vereinigten Staaten von Amerika-AWV-Shriftenreihe. s. 36, 37.
} 
tungen der Dienststellen bewertet, in der Regel auf Grund von Abteilungserfolgsrechnungen." ${ }^{21}$ Schmalenbach stellt die pretiale Betriebslenkung der bürokratischen Lenkung gegenüber und versucht, die Vorteile einer pretialen Betriebslenkung, die in der Praxis kaum zu finden war, darzustellen und damit die Unternehmer zu veranlassen, darüber nachzudenken, ob die Durchführung der pretialen Betriebslenkung nicht vorteilhafter wäre. Das Wort "pretial" kommt von lat. Pretium=Preis, und pretiale Lenkung bedeutet damit die Lenkungsfrom, die als Mittel Kostenund Erlöspreise braucht. Die bürokratischen Mittel sind dagegen Vorschriften und Anweisungen von verschiedener Art. ${ }^{22}$

Bei der pretialen Betriebslenkung haben die Abteilungsleiter einen großen Entscheidungsspeielraum. Sie können in weitem Umfange tun, was
sie wollen, aber sie müssen am Ende der Periode ein gutes Ergebnis vorlegen. $\mathrm{Da}$ manche Abteilungsgewinne nicht im Sinne des Gesamtbetriebes Gewinne darstellen, ist die Wahl der Verrechnungspreise von größter Bedeutung.

Die Anwendung der pretialen Betriebslenkung, wenn sie wirksam sein soll, ist an folgende Bedingungen geknüpft:

1) Die Abteilungsleiter müssen an den Ergebnissen ihrer Abteilungen interessiert sein.

2) Die Bildung und Gliederung der Abteilungen soll den Bedürfnissen der pretialen Betriebslenkung folgen.

3) Die obersten Instanzen dürfen sich nicht immer in die Angelegenheiten der Abteilungsleiter einmischen.

4) Die Verrechnungspreise müssen den Bedingungen der "optimalen Geltungszahl" entsprechen. ${ }^{23}$

5) Die dezentrale Organisation verlangt eine straffe Zentralisation des Rechnungswesens. Die Abteilungsleiter dürfen auf die Beobachtung ihrer Leistungen keinen Einfluß haben. "Es wäre ein Unding, den Werksleitern selbst das Werkzeug in die Hand zu geben, mit dem ihre Tätigkeit gemessen wird."24 Wenn diese Bedingungen erfüllt sind, kann die Anwendung der pretialen Betriebslenkung sehr nützlich sein.

${ }^{21}$ Schmalenbach, E.: a.a.O., s. 8.

22 Bender, K.: Pretiale Lenkung, s. 5, $\theta$.

23 "Die optimale Geltungszahl ist die Zahl, die wir bei verschiedenen Gütern ansetzen müssen; um angesichts der Vielfalt der Güter die richtige Entscheidung treffen zu können Richtige Entscheidung ist diejenige Zahl, die den höchsten Grad an Wirtschaftlichkeit verspricht. "Schmalenbach, E.: Pretialo Wirtschaftslen. kung. Bd. I Die optimale Geitungszahl. s. 14.

${ }^{24}$ Schmalenbach, E.: a.a.O., S. 13. 
Die pretiale Betriebslenkung hat folgende fünf Komponenten:2s
- a) Dezentralisation durch Verselbständigung der Abteilungen
b) Abteilungsergebnisrechung
c) Abteilungstantieme, mit der Zielsetzung der
d) Einführung unternehmerischer Geistes und der .
e) Förderung des Wirtschaftlichkeitsdenkens.

Zu (a): Die Dezentralisation gehört zum Wesen und zur Funktionsfähigkeit der pretialen Betriebslenkung. $\mathrm{Da}$ das Phänomen Dezentralisation schon eingehend erläutert wurde, wird es hier nicht wieder behandelt.

$\mathrm{Zu}$ (b): Die Abteilungsergebnisrechnung ist eine notwendige Bedingung für die pretiale Betriebslenkung, damit diese wirksam sein soll. Sie ist Vorausselzung für Abteilungstantiemen und ermöglicht weitgehende Dezentralisation. Sie erfüllt zugleich die Kontrollfunktion, die Kompaßfunktion und die Vergleichsfunktion, die zeitlich und auch zwischen Abteilungen erfolgen kann.

$\mathrm{Zu} \mathrm{(c):} \mathrm{Die} \mathrm{Abteilungstantieme} \mathrm{ist} \mathrm{nicht} \mathrm{im} \mathrm{gleichen} \mathrm{Umfang} \mathrm{not-}$ wendig wie die Abteilungsergebnisrechnung für die pretiale Betriebslenkung. Ihre Funktion kann auch auf anderen Wegen erreicht werden. "Sicher aber ist sie von diesen verschiedenen Möglichkeiten die gegenwärtig wirksamste und schmiegsamste."."

Zu (d): Die Wirtschaftsverfassung eines Landes, wie Schmalenbach sagt, ist von der Anwendung der pretialen Lenkung in hohem Grade abhängig. "Wenn es gelänge, in den Betrieben mehr als bisher die pretiale Betriebslenkung zur Anwendung zu bringen, würden der Bürokratengeist absterben und damit würde dann der freien Wirtschaft wiederum der Unternehmergeist zugeführt, dessen sie zu ihrer Existenz bedarf."27 Die Anwendung der pretialen Lenkung erfordert also das Vorhandensein des Unternehmersinns in einem großen Teil der Bevölkerung.

$\mathrm{Zu}$ (e): Wirtschaftlichkeitsdenken ist ein Hilfsmittel für die ökonomische Wertung des Handelns. Die pretiale Betriebslenkung fordert vondden Abteilungsleitern zugleich das Kosten- und Erlösdenken, also das Wirtschaftlichkeitsdenken. ${ }^{28}$.

\footnotetext{
25 Bender, K.: a.a.O., s. 9.

${ }^{26}$ Bender, K.: a.a.O., s. 53.

27 Schmalenbach, E.: a.a.O., s. 11.

28 Bender, K.: a.a.O., s. 8.
} 
Die Vorteile der pretialen Betriebslenkung: ${ }^{29}$

1) Der Verwaltungsapparat ist hier im Regelfall kleiner.

2) Das Wertungsdenken im Betriebe ist gefordert.

3) Da die nachgeordneten Betriebsleiter wesentlich größere Freiheit in ihrem Handeln haben, müssen sie die besten Mittel und Wege zur Erfüllung ihrer Aufgaben selbst suchen. Dadurch werden die obersten Instanzen Arbeitskraft und Zeit für wichtigere Arbeiten sparen können.

4) Weil die unteren Abteilungsleiter den Dingen und Menschen, über die zu disponieren ist, noch näher stehen, können sie den Leer- und Fehllauf sofort sehen und die notwendigen Maßnahmen ohne Zeitverlust ergreifen.

5) Das Prinzip der Mannigfaltigkeit

Die Nachteile der pretialen Betriebslenkung: ${ }^{30}$

1) Ein Nachteil der pretialen Betriebslenkung ist, da $\beta$ die unteren Abteilungsleiter leicht unbequeme Untergebene werden können. Das wiederum würde die Schwierigkeiten einer ohnehin schwachen Oberleitung noch erhöhen.

2) Es kann leicht zwischen den Abteilungsileitern zu Abrechnungsstreitigkeiten kommen.

3) Aus der Gesamtverantwortung der obersten Leitung ergibt sich immer eine Tendenz zur Zentralisation, also zur bürokratischen Lenkung.

\section{Die Suche nach einer optimalen Kombination von Zentralisation und Dezentralisation.}

Die Frage wird so lauten: wie sollen die Leitungsaufgaben auf die verscheidenen Stufen verteilt werden? Es ist klar, ehe eine Aufgabe ausgeführt wird, müssen alle ihre Merkmale mit ihren höchstmöglichen Genauigkeitsgraden bestimmt werden. Muß die oberste Leitung alle Merkmale selbst bestimmen oder mu $\beta$ sie auf die Bestimmung einiger oder sogar aller Merkmale verzichten und sie nach unten verlagern? Alle Genauigkeitsgrade können gar nicht von der obersten Leitung bestimmt werden. Selbst die ausführenden Aufgabenträger bestimmen einen groß̧en Bereich von Genauigkeitsgraden einer Tätigkeit, manchmal sogar bestimmen sie einige Merkmale selbst-.

29 Schmalenbach; E.: a.a.O., s. 15.

${ }^{30}$ Schmalenbach, E.: a.a.O., s. 16. 
Nach diesen Ausführungen ist klar geworden, da $\beta$ in einer Organisation Zentralisation und Dezentralisation immer gemischt vorhanden sind und vorhanden sein müssen. Das Problem liegt darin, die richtige Kombination von beiden zu finden. Optimal wird natürlich diejenige Kombination sein, welche die größtmögliche Wirtschaftlichekit gewährleistet. Wie diese Kombination zu erreichen ist, kann nur unter konkreter Umständen beantwortet werden.

Mit folgenden Worten son Simon werde ich meine Arbeit abschließen: "Insgesamt abschließend kann. man sagen, da $\beta$ sicherlich ein gewisses $\mathrm{Ma} \beta$ an Zentralisation unerläßlich .ist, wenn man die Vorteile der Organisation-Koordination, Erfahrenheit und Verantwortlichkeit erhalten will. Auf der anderen Seite darf man die Kosten der Zentralisation nicht außer acht lassen. Keinesfalls darf man Entscheidungen in die Hand der hochbezahlten Personen legen, die durch geringes bezahltes Personal spielend erledigt werden können. Zentralisation darf nicht zu einer Funktionsverdoppelung führen, die den Untergebenen überflüßig macht und das Mitteilungssystem beschwert. Und schließlich ist zu bedenken, da $\beta$ Zentralisation an die Stelle des höchst wirksam koordinierenden menschlichen Nervensystems einen zwischenpersonal koordinierenden Mechanismus setzt." ${ }^{\prime 1}$

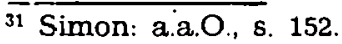

